\title{
Construction of a 3D Volumetric Probabilistic Model of the Mouse Kidney from MRI
}

\author{
Hirohito Okuda ${ }^{1,2,3}$, Pavel Shkarin ${ }^{3}$, Kevin Behar ${ }^{4}$, James S. Duncan ${ }^{2,3}$, and \\ Xenophon Papademetris ${ }^{2,3}$ \\ ${ }^{1}$ Production Engineering and Research Lab., Hitachi Ltd., Kanagawa, Japan, \\ ${ }^{2}$ Department of Biomed. Engineering, \\ ${ }^{3}$ Diag. Radiology and ${ }^{4}$ Phsychiatry \\ Yale University New Haven, CT 06520-8042
}

\begin{abstract}
We present the results of constructing a probabilistic volumetric model of 3D MR kidney images. The ultimate goal of this work is the mouse kidney segmentation based on a probabilistic volumetric model. The kidneys were aligned into the base shape using an extended robust point matching algorithm. The registration step consists of the global linear transformation and the local B-spline based free form deformation. Shape modeling is performed with globally aligned shape and template volumetric image is generated with locally aligned images. We are currently working on developing a segmentation algorithm using our model.
\end{abstract}

\section{Introduction}

The ultimate goal of this work is to automate the segmentation of kidneys, and to quantify kidney volume in transgenic mouse models [1] of polycystic kidney disease. Toward this goal, here we present the result of constructing a probabilistic volumetric model which is the first key component of our strategy for the segmentation process. A general drawback of the model constructing methods proposed so far $[2,5]$ is the correspondence problem where the definition of one-to-one mapping across data are needed. To solve this problem, we apply the extended robust point matching algorithm (RPM)[3] which can automatically compute the correspondences. Here we present a result of probabilistic volumetric model of both kidneys constructed using RPM .

\section{Method and Results}

Figure 1(a) shows an example of training image data. Ten postmortem eight-week old C57BL6 wild type mice were scanned. All imaging was performed on a Bruker $4.0 \mathrm{~T} / 40 \mathrm{~cm}$ bore animal system using a T2-weighted 3D Multi-spin multi-echo sequence $(\mathrm{MSME})$, with a $\mathrm{TE}=15 \mathrm{~ms}, \mathrm{FOV}=4 \times 2.5 \times 1.8 \mathrm{~cm}$ and an imaging matrix of $256 \times 128 \times 64$.

An expert user performed the original surface extraction of both kidneys from the images with a software platform originally designed for segmenting the left ventricle 
of the heart. Segmented surfaces are aligned into the common base shape with RPM, first by a global linear transformation and next, by a local B-spline based free form deformation[4]. An example of this registration process is shown in Figure 1. The target kidney in Figure 1(b) is aligned into the base shape with a global linear transformation. The shape difference which remains after the linear transformation (Figure 1(c)) shows the shape variation to be modeled that is invariant to affine transformation. The final result of the registration after the local free form deformation (Figure 1(d)) indicates the RPM registration is enough accurate to construct an intensity model on the base shape. This yields an explicit volumetric deformable model as opposed to a model of simple surface shape.

The free form deformation is parameterized by concatenating the displacement vectors on grid points, and is used as an implicit representation of the kidney shape. The shape modeling is done by performing principal component analysis (PCA). The mean shape and first three modes of PCA are shown in Figure 2 (a) and (c). The modes of model are sorted in decreasing magnitude of their corresponding eigenvalues. The result shows the large variability included in the kidney model. Also, the template volumetric image is generated by transforming training data set into the base shape and averaging the transformed training data (Figure 2 (b)). This volumetric template image with statistical shape model could be used as the probabilistic volumetric model in the segmentation process.

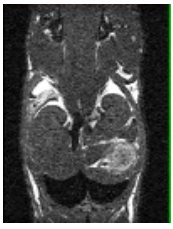

(a) Training data

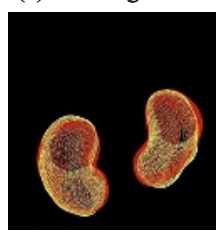

(c) Linear

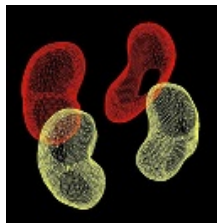

(b) Before alignment

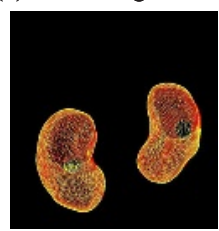

(d) FFD

Fig. 1. Model construction step. (red) target, (yellow) base

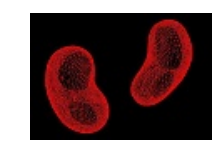

(a) mean shape

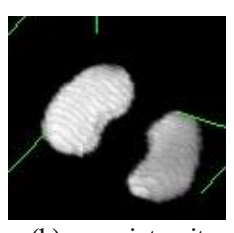

(b) mean intensity

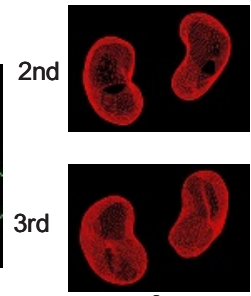

$-2 \sigma_{i}$
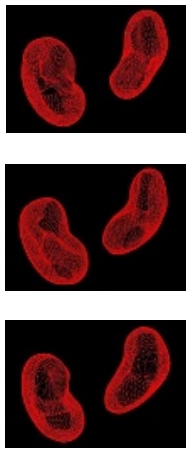

$2 \sigma_{i}$

(c) PCA

Fig. 2. Result of Model construction

\section{Conclusion}

We demonstrate the feasibility of applying the RPM registration technique for the construction of probabilistic volumetric model of mouse kidney. We are currently working on developing a segmentation algorithm using the model proposed here.

Acknowledgments. We would like to thank Production Engineering Research Lab., Hitachi Ltd. for giving the opportunity of this research. 


\section{References}

1. G.Wu et al, Somatic inactivation of PKD2 results in polycystic kidney disease. Cell, 93:177- 188, 1998

2. T.F. Cootes, C. Beeston, G. Edwards, and C. Taylor : Unified framework for atlas matching using active appearance models. IPMI, (1999).

3. Xenophon. Papademetris, Andrea P. Jackowski. Robert T. Schultz, Lawrence H. Staib, James S.Duncan : Computing 3D Non-rigid Brain Registration Using Extended Robust Point Matching for Composite Multisubject fMRI Analysis. MICCAI2003, 788-795.

4. Daniel Rueckert, Alejandro F. Frangi, Julia A. Schnabel : Automatic Construction of 3-D Statistical Deformation Models of the Brain Using Nonrigid Registration, IEEE Transactions On Medical Imaging,vol.22,No.8.Aug. 2003.

5. S. S. Gleason, H. Sari-Sarraf, M. A. Abidi, O. Karakashian, and F. Morandi. A new deformable model for analysis of X-ray CT images in preclinical studies of mice for polycystic kidney desease. IEEE Transactions on Medical Imaging, 21(10):1302-1309, October 2002. 